\title{
Giving words to emotions: the use of linguistic analysis to explore the role of alexithymia in an expressive writing intervention
}

\author{
Alesia Renzi, Rachele Mariani, Michela Di Trani, Renata Tambelli \\ Department of Dynamic and Clinical Psychology, "Sapienza” University of Rome, Rome, Italy
}

\begin{abstract}
Expressive writing techniques are methods focusing on written emotional expression that require people to write about traumatic or difficult experiences, with the objective of promoting an elaboration of these events. The general aim of the study is to investigate the influence of alexithymia, a deficit in emotional regulation processes, on the effects of an expressive writing intervention, analyzing the writing protocols through the use of the Linguistic Inquiry and Word Count (LIWIC) and Referential Process (RP) linguistic measures via IDAAP software. Thirty-five women undergoing an assisted reproductive treatment participated in the study and filled out a sociodemographic questionnaire, the 20-item Toronto Alexithymia Scale. They also underwent three session of writing, following a request that they write about their emotions regarding their current situation. The women enrolled were divided into two groups: low alexithymia and high alexithymia, comprising individuals with a TAS-20 total score lower or higher than the mean, respectively. Analyses within the groups during the three writing sessions revealed that the women with low alexithymia reported a greater number of words expressing affectivity, sadness and future perspective, whereas no significances in the high alexithymia group emerged. Moreover, when analysing differences between the groups, high-alexithymia women reported lower scores in RP indexes and fewer words expressing sadness, future perspectives and we verbal. In conclusion, these preliminary findings may confirm the hypothesis that alexithymia affects the effectiveness of expressive writing through a difficulty in becoming involved in the writing process and a lack of symbolizing processes.
\end{abstract}

Key words: Expressive writing intervention; alexithymia; multiple code theory; linguistic measures.

Correspondence: Alessia Renzi, Department of Dynamic and Clinical Psychology, "Sapienza" University of Rome, via degli Apuli 1,00185 , Rome, Italy.

Tel.: +390649917989.

E-mail: alessia.renzi@uniroma1.it

Citation: Renzi, A., Mariani, R., Di Trani, M., \& Tambelli. R. (2020). Giving words to emotions: the use of linguistic analysis to explore the role of alexithymia in an expressive writing intervention. Research in Psychotherapy: Psychopathology, Process and Outcome, 23(2), 121-132. doi: 10.4081/ripppo.2020.452

Ethical approval: All procedures performed in studies involving human participants were in accordance with the ethical standards of the institutional and/or national research committee and with the 1964 Helsinki declaration and its later amendments or comparable ethical standards. Ethical approval was granted by the Ethics Committee of the Department of Dynamic and Clinical Psychology of the University of Roma "Sapienza".

Conflict of interest: the authors declare no potential conflict of interests.

Received for publication: 7 February 2020.

Accepted for publication: 21 April 2020.

This work is licensed under a Creative Commons Attribution NonCommercial 4.0 License (CC BY-NC 4.0).

${ }^{\circ}$ Copyright: the Author(s), 2020

Licensee PAGEPress, Italy

Research in Psychotherapy:

Psychopathology, Process and Outcome 2020; 23:121-132

doi:10.4081/ripppo.2020.452

\section{Introduction}

\section{Expressive writing interventions and the alexithymia construct}

Expressive Writing Interventions (EWI) are methods focusing on written emotional expression that originally required people to write about past traumatic experiences, with the objective of promoting an elaboration of these events (Pennebaker, 1997; Solano, 2013). In subsequent studies, the writing topics were expanded to other specific difficult experiences, obtaining beneficial effects in different healthy and clinical populations (Di Trani, Renzi, \& Solano, 2014; Renzi et al., 2019). There remain no standard instructions for EWI. Rather, the topic can be formulated depending on the specific subjects to whom it is addressed, with the additional possibility of proposing instructions focused on a present difficult experience and dedicated to positive expectations for the future, utilizing a different writing topic for each session (Di Trani et al., 2014).

In general, the EWI protocol asks subjects to write about their deepest thoughts and feelings concerning a present or past difficult event across three or four writing sessions (Pennebaker, 1997). The effectiveness of expressive writing represents a complex phenomenon; in general, EWI seems to promote a reflection and re-evaluation of an experience. The growing number of studies on EWI has made it possible to highlight several possible explanations - which are not mutually exclusive - that may illustrate the mechanisms underlying the effectiveness of 
expressive writing (Pennebaker \& Chung, 2007; Solano, 2013). It has been suggested that the mere act of writing requires a certain ability to structure a coherent narrative, reflect on an event, recognize causal connections and acknowledge the emotions involved that must be translated into words (Pennebaker \& Chung, 2007; Solano, 2013). This process promotes a different representation of the events in the memory; indeed, the memory is simplified and can be easily recalled to the mind. These cognitive changes imply a different understanding of the experience as well as a change of perspective on the event (Pennebaker \& Chung, 2007).

In some ways, EWI may be considered an expressive and focal psychotherapy (Solano, 2013), as several phenomena that have been used to explain the effectiveness of EWI have also been used to explore the effectiveness of psychodynamic psychotherapy processes, such as by structuring a more aware narrative and acknowledging and decoding one's emotions into words. It must be specified that it is an analogue and not the same as psychotherapy. Moreover, the main differences are the technique and the subjects to whom the intervention is addressed: in a psychotherapy setting, people ask for a clinical intervention, whereas the EWI is usually proposed for populations that have not requested psychotherapy but are deemed to be exposed to stressful events (Solano, 2013).

In this respect, one should not create expectations among the subjects participating in an EWI that they will receive an advantage, as it is important to avoid shifting the request/need on the subjects. Instead, it is prudent to maintain a research framework in which the request/need is attributed to the researcher. It can be easier to establish a good supportive relationship starting from the idea of doing research than starting from the idea of doing therapy, as in this way the relationship appears more equal: researchers do not ask people to recognize that they have a need; the subjects are the experts while the researchers are the ones who need data (Solano, 2013). This position has been reinforced by several studies that have shown that when the writing intervention is presented as something that will be useful to the subject, the refusal rate to participate is greater, providing evidence of people's difficulty in taking full responsibility for a psychological intervention in the absence of a personal request (Di Trani et al., 2014; Solano, 2013; Spera, Buhrfeind \& Pennebaker, 1994). The absence of a clear request can represent both a limitation and a strength of the technique, allowing researchers to propose a psychological intervention to several contexts in which people are facing a difficult experience, thereby configuring the EWI as a health promotion intervention (Di Trani et al., 2014).

In this direction, EWI can represent an appropriate intervention in women undergoing an Assisted Reproductive Treatment (ART), with the aim of promoting psychophysical health in a stressful condition. Certainly, it may support women in monitoring their internal status and in acquiring greater ability in the cognitive-emotional processing of the experience. Both infertility and its treatments are considered a stressful experience, with serious implications for individuals' mental, physical and social well-being (Ben Shlomo, Skvirsky, Taubman-Ben-Ari, Azuri, \& Horowitz, 2016; Galhardo, Cunha, \& PintoGouveia, 2011). Accordingly, the provision of psychosocial interventions for people facing these experiences has been recommended by regulatory bodies in several countries, by various associations involved in the care of infertile people and by several mental health professionals working with infertile couples (Boivin, 2003; Renzi et al., 2019).

The effects of EWI in infertility subjects undergoing an ART have been examined in previous studies but have yielded contrasting results. For instance, Matthiesen et al. (2012) found EWI effective in reducing infertility-related stress, Frederiksen et al. (2017) found EWI effective in reducing depressive symptoms but not in promoting pregnancy, Renzi et al. (2019) found EWI effective in promoting pregnancy and in reducing alexithymia and psychophysical symptoms, while Panagopoulou, Montgomery and Tarlatzis (2009) obtained no significant results in terms of stress and pregnancy rates. In fact, despite the large number of promising studies, the overall effect size of writing interventions is modest in this field. This may owe to the fact that several individual differences can influence the success of the writing technique, playing a role in people's capacity to use the intervention effectively (Di Trani, 2007). In this direction alexithymia, literally meaning 'a lack of words to express emotion' (Nemiah, Freyberger, \& Sifneos, 1976), has been conceptualized as an emotional dysregulation disorder, characterized by difficulties in identifying and expressing emotions, in employing fantasy to regulate painful feelings and to find creative solutions to problems as well as in communicating needs to others in order to obtain support (Taylor, 2004; Taylor \& Bagby, 2013). Therefore, alexithymia represents a vulnerability factor and is associated with both physical and mental disorders (Gourounti, Anagnostopoulos, Griva, \& Vasmatzis, 2016; Grabe et al., 2008; Luminet, Bagby \& Taylor, 2018; Taylor, 2004). However, few studies specifically regarding the role of alexithymia in infertility have been conducted to date. Most investigations concerning this specific topic have compared infertile and fertile subjects and have concluded that alexithymia levels are significantly higher among the former (Conrad, Schilling, Langenbuch, Haidl, \& Liedtke, 2001; Lamas et al., 2006; Moreno-Rosset, del Castillo Aparicio, Ramírez-Uclés, \& Martín Díaz, 2011; Shi, Wu, $\&$ Zhu, 2006). A small number of studies have explored the relationship between alexithymia and psychopathological dimensions, identifying a positive correlation between alexithymia levels and emotional maladjustment to infertility (Ramírez-Uclés, Castillo-Aparicio, \& MorenoRosset, 2015), anxiety symptomatology (Shi et al., 2006), 
coping strategies and stress (Gourounti et al., 2016). Moreover, only one study has identified a significant role of alexithymia as a predictor of infertility-related stress (Dumitru \& Turliuc, 2015). In conclusion, considering the importance of having optimal contact with one's emotions and needs in order to deal with distress, alexithymia may influence the psychophysical well-being of people facing ART. Initially Taylor, Bagby and Parker (1997) proposed that alexithymia reflects a deficit in the cognitive processing of the emotions involved in the elaborative process promoted by an EWI and, more generally, by different psychotherapy programmes. A lack of imagination, psychological mindedness and awareness of one's emotions may play a significant role in reducing one's ability to successfully engage in psychotherapy (Grabe et al., 2008; Luminet et al., 2018; Taylor et al., 1997; Porcelli et al., 2003). In fact, during dynamic psychotherapy, the capacity to symbolize and translate emotions into language is often considered to be critical for improving symptoms. These capacities are greatly reduced or lacking in alexithymia (Messina, Beadle, \& Paradiso, 2014).

In this direction, studies specifically investigating the beneficial effects of expressive writing in individuals with different alexithymia levels have attained contrasting results. Some studies have shown that high-alexithymia individuals tend to benefit from writing more than their low-alexithymia counterparts (Paez, Velasco, \& Gonzalez, 1999; Pennebaker \& Chung, 2007; Solano, 2013; Solano, Donati, Pecci, Persicheeti, \& Colaci, 2003), whereas in others, alexithymic subjects have not appeared to reap the same benefits after writing (Lumley, 2004; Pennebaker \& Chung, 2007; Solano, 2013).

As regards the only study concerning the effectiveness of EWI in reducing alexithymia levels and psychophysical symptoms as well as in promoting pregnancy, no significant effects were found in terms of psychological dimensions: indeed, the only significant result was related to the promotion of positive outcomes in ART treatments (Renzi et al., 2019). Furthermore, the median value of the total alexithymia scores of both experimental and control groups was used to create four subgroups of women (experimental low/high alexithymia and control low/high alexithymia) in order to jointly evaluate the effects of treatment and alexithymia on pregnancy rates (Renzi et al., 2019). Statistically significant differences between the four subgroups did not emerge $(p=.09)$, although it was qualitatively interesting to note that in the experimental group, the ART rate of success increased from $22.7 \%$ in the high-alexithymia subgroup to $33.3 \%$ in the low-alexithymia subgroup (Renzi et al., 2019). This finding, although not statistically significant, may support the hypothesis of a different activation of the elaborative process promoted by the EWI according to different alexithymia levels. This renders it necessary to conduct investigations into the texts produced by the writing subjects. In fact, according to Pennebaker and Chung (2007), the degree to which individuals are able to cognitively organize an event into a coherent narrative is a marker that the specific experience has achieved a knowledge status. In some cases, through analyzing the language that people use, it is possible to determine the degree to which people have come to know their emotions and experiences.

The Linguistic Inquiry and Word Count (LIWC; Pennebaker, Booth, \& Francis, 2007), a computerized text analysis programme, was initially created to analyze essays from EWI studies, although it is also routinely used to analyze psychologically meaningful writing (Pennebaker, Mehl, \& Niederhoffer, 2003; Tausczik \& Pennebaker, 2010). The programme includes analyses of negative and positive emotional words, causal words and insight words. The LIWC programme has also been used to explore associations between word usage and various health and behavioural outcomes in expressive writing subjects (Pennebaker, Mayne, \& Francis, 1997). Through the use of this programme several findings, have emerged: i) the more people use positive emotion words, the more their health improves; ii) negative emotion word use is curvilinearly related to health change after writing; iii) improvements in the use of causal and insight words is related to improvements in health and working memory; iv) the more people alter their use of function words during writing sessions, the more their health improves; and v) the more people oscillate in their use of first-person singular pronouns and all other personal pronouns, the more their health improves (Campbell \& Pennebaker, 2003; Klein \& Boals, 2001; Pennebaker \& Chung 2007; Petrie, Booth, \& Pennbaker, 1998; Rohrbaugh, Shoham, Skoyen, Jensen, \& Mehl, 2012). In a recent study, LIWIC was applied to written descriptions of life experiences, revealing that alexithymia is associated with a negative bias in emotional experience and a hyper-focus on the self (Edwards, Shivaji, Micek, \& Wupperman, 2020).

In conclusion, the role of alexithymia in EWI still represents an open field of research and investigation of the texts that people produce during writing sessions using LIWIC may help develop a deeper understanding of its role in the writing process.

\section{The emotional blindness of alexithymia from a multiple code theory perspective}

Taylor and Bagby (2013) have suggested that alexithymia may be more fully understood in the light of Bucci's (1997a) Multiple Code Theory (MCT). MCT is a general theory of emotional information processing, derived from current work in cognitive psychology, psychoanalysis and affective neuroscience (Bucci \& Maskit, 2007). According to Bucci (1997a; 2001), humans use three basic ways to elaborate information and build up images and representations: subsymbolic, non-verbal symbolic and verbal symbolic processing systems. In the subsymbolic system, multiple forms of information are processed simultaneously in a comprehensive and analog- 
ical modality, in addition to continuous dimensions, which organize the affective core. This system is implicated in recognizing non-verbal communication, such as identifying a familiar voice, doing physical exercises, or performing creative work. The non-verbal symbolic system works with discrete images or representations that take shape from the continuous process of subsymbolic experience. Lastly, in the verbal symbolic system, these images and representations can be decoded into words. Any recoding process from one system to another cannot involve all material, because any system maintains its own specificity and works in parallel. Bucci (1997a) has proposed that the three types of processing systems are interconnected by a Referential Process (RP), a set of functional and bidirectional stages representing the process of linking non-verbal material into a form that can be communicated to others through language (Bucci, 1997a, 2001; Bucci, Maskit, \& Murphy, 2015). RP allows individuals to communicate emotional experiences to others and to perform a function of self-mutual emotional regulation. When the RP is interrupted (e.g. via a specific internal conflict or trauma), the verbal and non-verbal systems within the emotion schemas become disconnected. This induces a block in the organization of the schemas, in self-emotional regulation and in the construction of meanings of affective experience (Mariani, Di Trani, Negri, \& Tambelli, 2020). The idea of disconnection within the emotion schemas can be applied to alexithymia: "without linkages to images and words, the individual is unable to symbolize emotional states" (Taylor \& Bagby, 2013; p. 105). Several studies have highlighted that alexithymia is associated with deficits in labelling emotional facial expressions, with reduced perceptual abilities, with impaired semantic representations of emotional concepts and with neither specific emotional facial expressions nor a specific valence (Grynberg et al., 2012; Pedrosa Gil et al., 2009; Ridout, Thom, \& Wallis, 2010). Moreover, a recent study by Di Trani, Mariani, Renzi, Greenman and Solano (2018) has revealed a relationship between alexithymia levels and several indexes related to RP in the general population. By contrast, no studies have explored the connections between RP and alexithymia in clinical process, such as expression writing technique, in order to examine several implications of alexithymia for clinical treatments. It can be assumed that the emotional difficulties involved in the alexithymia dimension will preclude a full connection between emotions and words. People with higher alexithymia levels may not be able to use the writing process in the same way as people with lower alexithymia levels, precisely because the dimension of emotional disconnection between subsymbolic and symbolic experiences is greater. Qualitatively investigating the relationship between alexithymia, RP and EWI would facilitate a greater understanding of how to develop and propose an intervention that can be effective and useful for different populations.

The main aim of the present study is to investigate the possible influence of alexithymia on people's capacity to use EWI as a supportive clinical intervention during ART, through a linguistic analysis conducted by two different computerized linguistic analysis systems (LIWIC and RP linguistic measures), processed by the Italian Discourse Attribute Analysis Program (IDAAP). The more specific aims are:

a) to explore possible differences within groups with different levels of alexithymia in both linguistic measure systems (LIWC and RP) across three writing sessions;

b) to explore possible differences between groups with different levels of alexithymia in LIWC and RP indexes. Specifically, regarding RP, we hypothesize that women scoring higher in terms of alexithymia will exhibit more disconnections among systems and hence no activation of the symbolizing phase or the reflection/reorganization phase. Regarding LIWC, we hypothesize that women scoring higher in terms of alexithymia will show a higher use of negative emotional words, words focusing on the self and words focusing on the present. A lower use of positive emotional words, a lower sense of "we-ness" (use of pronoun "we" and "we verbal") and fewer words focusing on the future are also hypothesized, reflecting a difficulty in using fantasy to imagine pregnancy and parenthood.

\section{Materials and Methods}

\section{Participants}

The participants comprised women undergoing an ART treatment who were being consecutively admitted to a specialized centre in Rome. The women were selected according to the following inclusion criteria: i) Undergoing an in vitro fertilization treatment with embryo transfer (IVF-ET) or intra-cytoplasmic sperm injection (ICSI); ii) Being at the first medical visit before starting a given cycle of ART treatment; iii) Performing all medical checkups at the centre in order to have the opportunity to complete the writing protocol; iv) Undergoing the ART treatment for a fertility problem, not for a pre-implantation genetic diagnosis (PGD).

We excluded women with a history of psychiatric disorder, who were unable to understand and/or write in Italian and/or had already participated in the study in a previous ART cycle.

In total, 42 women were invited to participate in the study: 35 women agreed to participate and completed the EWI protocol, one woman was not included in the investigation due to difficulties in writing in Italian, while six declined. The participants were living in Rome or nearby, since the medical request to perform follicular monitoring and blood samplings every other day. The women reported a mean age of 36.17 years $(\mathrm{sd}=5.067)$. Three per cent reported an educational level of eight years, $40 \%$ of 13 years and $57 \%$ of 16 years. Eighty-three per cent of the participants were married whereas the other $17 \%$ were 
cohabiting. In terms of employment status, $57 \%$ of the women were employee, $26 \%$ were freelance, $14 \%$ were unemployed and $3 \%$ were homemakers.

\section{Measures}

\section{Socio-demographic questionnaire}

The questionnaire was designed to collect information concerning age, social status, education level and occupational activity.

\section{The 20-Item Toronto Alexithymia Scale} (TAS-20; Bagby, Parker, \& Taylor, 1994; Bressi et al., 1996)

The TAS-20 is structured according to three factors: difficulty in identifying feelings (F1), difficulty in describing feelings (F2) and externally oriented thinking (F3). Each item is rated on a five-point Likert scale, ranging from 'strongly disagree' (1) to 'strongly agree' (5). This instrument provides both a total score and a score for each factor. Total scores range from 20 to 100 . The questionnaire showed adequate internal reliability (total score of Cronbach's $\alpha=.75$ ) and test-retest reliability $(r=83)$. A total score of Cronbach's $\alpha$ of .79 was obtained in the present study.

\section{Computerized linguistic measures of a referential process}

Historically, an RP consisted of hand-coding narratives along specific dimensions (Bucci, Kabasakalian, \& the Referential Activity Research Group, 2004), but more recently a computerized programme has been developed to allow for automated coding by comparing speech with specific linguistic dictionaries: the IDAAP (Maskit, Bucci, \& Murphy, 2012; Maskit \& Murphy, 2011). The Discourse Attributes Analysis Program (DAAP) is a computer-based text analysis system designed by Maskit (2014), whose features include the use of both weighted and unweighted dictionaries and whose output includes measures based on an exponential smoothing operator. In our study, we chose the following dictionaries and derived measures built and validated for the Italian language in order to explore the RP in the writing process.

The Italian Weighted Referential Activity Dictionary (IWRAD; Mariani, Maskit, Bucci, \& De Coro, 2013) is a computerized measure of Referential Activity (RA) in the Italian language. It contains a list of 9,596 frequently used Italian words, each assigned a weight between 0 and 1, with 0.5 as the neutral value. A high score represents a high level of RA, corresponding to a high level of concreteness, specificity, clarity and imagery in the speech sample. Part of the value of the IWRAD derives from its power to assess linguistic style (rather than only focusing on content) and to represent the unintended aspects of emotional involvement. For a deeper discussion regarding the method of building the weighted dictionary, see Maskit et al. (2012), and Maskit and Murphy (2011). High scores on this measure detect and model the symbolizing phase of the RP as understood through MCT.
The Mean High-Italian Weighted Referential Activity Dictionary (MH-IWRAD; Mariani et al., 2013) is calculated on IWRAD scores and is defined as a referential activity intensity index (i.e. a measure of high intensity of emotional engagement) emerging from the speech. It indicates how high the IWRAD is when it is above the mean. It is obtained by looking only at words with IWRAD scores lying above the neutral value and then computing for these words only the average amount of the IWRAD scores. This is perhaps best understood as a measure of upward oscillations in the RA scores.

Italian Weighted Reflection and Reorganization List (IWRRL; Negri et al., 2018): The reorganization and reflection function refers to the degree to which the speaker is trying to recognize and understand the emotional significance of an event or set of events in their own or someone else's life, or in a dream or fantasy. It is not about abstract reflection but rather a person's reasoning related to an experience that has been vividly experienced. The IWRRL is a list of Italian weighted (ranging from 0 to 1 , with 0.5 as the neutral value) words referring to the reorganization and reflection function; the list consists of 1,633 words with $89 \%$ coverage of the Italian language as it normally spoken (Negri et al., 2018). High scores on this measure represent high reflection/reorganizing as understood through MCT. Through IWRRL, it is possible to detect and model the reorganizing.

The Mean High-Italian Weighted Reflection and Reorganization List (MH-IWRRL; Negri et al., 2018) is calculated on IWRRL scores and is a measure of the high intensity of the reflection and reorganization function as emerges from a person's speech. It indicates how high the IWRRL is when it is above the mean. It is obtained by looking only at words with IWRRL scores lying above the neutral value and then computing for these words only the average amount of the IWRRL scores. It is best understood as a measure of upward oscillations in the reflection and reorganizing scores.

Covariation between IWRAD and IWRRL (IWRAD_IWRRL; Negri et al. 2018) is a derived measure as a function of smoothing measures over the course of a narrative. DAAP is able to calculate indexes of the extent to which any pair of measures tend to move together or move in opposite directions. This adds an entirely new dimension to text analysis and enables us to measure the extent to which IWRAD and IWRRL go together or in opposite directions. Higher scores show that symbolizing and reorganizing flow together, while lower scores indicate variables present the opposite pattern.

\section{Linguistic Inquiry and Word Count \\ (Pennebaker, Booth, \& Francis, 2015)}

The Linguistic Inquiry and Word Count (LIWC) is a computerized programme that seeks to analyze data related to language used in written reports. The progeamme includes the main text analysis module alongside a group 
of built-in dictionaries. LIWC reads written or transcribed verbal texts and then compares each word in the text against a user-defined dictionary. After the processing module has read and accounted for all the words in a given text, it calculates the percentage of total words that match each of the dictionary categories. LIWC2015 v1.6 software was used here together with Italian LIWC_2007 Dictionaries. Specific word categories were chosen for the purpose of this study: Pronouns I and we; I verbal and we verbal; affective words; positive emotions; optimism; negative emotions; anxiety; anger; sadness; past time; present time; future time.

\section{Procedure}

The study was conducted between January 2015 and January 2017. It was carried out in accordance with the code of ethics of the World Medical Association (Declaration of Helsinki) for experiments involving humans. Ethical approval was granted by our department's ethics committee.

The women were informed of the study by the gynaecologist responsible for the medical procedure during their first gynaecological visit in which the pharmacological treatment was established in order to begin the specific ART treatment. The gynaecologist ascertained women's eligibility for the study during the first clinical evaluation, these being the exclusion/inclusion criteria routinely explored in this phase. After the medical visit, the gynaecologist introduced the eligible women to the psychologist responsible for the research protocol's implementation. Here the psychologist deeply illustrated the research protocol and the women who agreed to participate signed an informed consent before filling out the tests. The entire research protocol took place in the medical centre and was implemented by a qualified psychologist in a specific room adjacent to the waiting room while the subjects were waiting for their medical appointments, which generally were every other day. The psychologist was present in the clinic every day the participants have the medical visit in order to personally assign the test and the writing materials (sheet of paper with writing instructions and a pen) as well as to receive the completed task, thereby maintaining an ongoing relationship with the participants. The setting was adequate to ensure both the women's privacy and the possibility of focusing on the writing task without distractions.

Three encounters were required of the participants: in the first, the women filled out the questionnaires and completed the first expressive writing session, whereas in the second and third they completed the expressive writing protocol. The writing instructions were based on guidelines developed by Pennebaker (1997) and adapted for this particular clinical population. The topic was the same for each writing session:

"For the next three sessions I would like you to write about your deepest thoughts and feelings about your ex- perience of the assisted reproduction technology treatment. In your writings I would like you to explore your deepest emotions and thoughts related to this experience. You can link your writings to relationships with others (including your partner, parents, friends and relatives), to your past, present or future, or to what you were, you would like to be or you are now, assuming that everything has proceeded well. Do not worry about the perfect use of the Italian language; the only rule is that once you start writing, you continue until the end of the time at your disposal (20 minutes)".

\section{Statistical Analyses}

All statistical analyses were performed using the Statistical Package for Social Science version 24 (SPSS version 24, Armonk, NY). Data are reported here as means and standard deviations for continuous variables and as percentages for discrete variables. According to the TAS20 mean total score obtained by the women enrolled in the study (=44.97), the subjects were divided into two groups: low alexithymia (comprising women reporting a TAS-20 total score lower than the mean) and high alexithymia (comprising women reporting a TAS-20 total score higher than the mean). One-way analyses of variance (ANOVAs) and chi-squared tests were performed in order to evaluate the homogeneity of the two groups of women for continuous and discrete variables, respecively. One-way ANOVAs were also performed in order to evaluate differences during the three writing sessions in linguistic measures of RP and LIWIC indexes within groups of women with low/high alexithymia scores and to evaluate differences in linguistic measures between groups of women scoring low/high in terms of alexithymia in the total written production. Bonferroni correction was applied to the post-hoc tests. A p value $<.05$ was considered significant.

\section{Results}

The 35 women enrolled reported a mean age of 36.17 years $(\mathrm{sd}=5.067)$ and a mean TAS-20 total score of 44.97 $(\mathrm{sd}=13.89)$. Using the TAS- 20 mean total score, the sample of women was divided into two groups under and over the alexithymia mean value: the low-alexithymia group comprised 21 women showing a TAS-20 mean score of $35.42(\mathrm{sd}=5.71)$, whereas the high-alexithymia group comprised 14 women showing a TAS-20 mean score of $59.63(\mathrm{sd}=9.07)$. The homogeneity between groups in terms of age, educational level and social and employment status was evaluated. No differences in age $(\mathrm{F}=.052$; $\mathrm{p}=.821)$, educational level $\left(\chi^{2}=.714 ; \mathrm{p}=.700\right)$, social status $\left(\chi^{2}=.134 ; \mathrm{p}=.714\right)$ or employment status $\left(\chi^{2}=1.042\right.$ $\mathrm{p}=.791)$ emerged.

Tables 1 and 2 report the results related to the different uses of the writing intervention during the three sessions 
within groups of subjects with low and high levels of alexithymia, respectively. The results indicated significant differences in the affectivity, sadness and future time indexes of LIWIC among the women with low levels of alexithymia (respectively $\mathrm{p}=.025 ; \mathrm{p}=.013 \mathrm{p}=.049$ ). Bonferroni's post hoc analysis revealed for both affectivity and sadness

Table 1. Differences in linguistic measures of the RP and LIWIC indexes during the three writing sessions with the low-alexithymia group.

\begin{tabular}{|c|c|c|c|c|c|c|c|c|}
\hline \multirow[t]{2}{*}{ Variable } & \multicolumn{2}{|c|}{ I writing session } & \multicolumn{2}{|c|}{ II writing session } & \multicolumn{2}{|c|}{ III writing session } & \multirow[t]{2}{*}{$\mathbf{F}$} & \multirow[t]{2}{*}{$\mathbf{p}$} \\
\hline & M & sd & M & sd & M & sd & & \\
\hline \multicolumn{9}{|l|}{ LIWIC } \\
\hline I & 3.6719 & 2.2075 & 4.1590 & 2.2920 & 3.4652 & 2.5581 & 0.480 & 0.621 \\
\hline We & 0.7395 & 0.7841 & $0-6495$ & 0.7822 & 0.5376 & 0.7957 & 0.347 & 0.709 \\
\hline Affectivity & 5.8314 & 2.4856 & 7.4014 & 2.4410 & 8.2314 & 3.4183 & 3.929 & $0.025^{*}$ \\
\hline Positive sensations & 3.4329 & 1.9600 & 4.4162 & 2.5141 & 4.5781 & 3.2013 & 1.186 & 0.313 \\
\hline Positive emotions & 1.0838 & 0.9856 & 1.2733 & 1.4741 & 1.0876 & 1.4229 & 0.143 & 0.867 \\
\hline Optimism & 1.2581 & 1.0447 & 1.9386 & 1.6402 & 2.2467 & 2.1326 & 1.935 & 0.153 \\
\hline Negative emotions & 2.3529 & 1.7496 & 2.9610 & 1.9910 & 3.6186 & 2.5395 & 1.875 & 0.162 \\
\hline Anxiety & 0.9862 & 1.3041 & 1.3300 & 1.6078 & 1.2943 & 1.3098 & 0.375 & 0.689 \\
\hline Anger & 0.3724 & 0.5761 & 0.4510 & 0.7201 & 0.02295 & 0.5503 & 0.688 & 0.506 \\
\hline Sadness & 0.3067 & 0.5042 & 0.7338 & 0.7733 & 1.1300 & 1.2005 & 4.457 & $0.013 *$ \\
\hline Past time & 2.1733 & 2.0097 & 1.1443 & 1.2259 & 1.5300 & 1.6796 & 2.036 & 0.139 \\
\hline Present time & 10.8424 & 3.8089 & 10. & 2381 & 2.9003 & 10.6138 & 3.1401 & 0.179 \\
\hline \multicolumn{9}{|l|}{0.837} \\
\hline Future time & 0.3924 & 0.7605 & 0.7452 & 0.7220 & 0.2190 & 0.5763 & 3.164 & $0.049 *$ \\
\hline I verbal & 4.8871 & 2.5660 & 3.8833 & 2.1429 & 4.8152 & 2.2619 & 1.212 & 0.305 \\
\hline We verbal & 0.9067 & 1.5576 & 0.9229 & 1.2566 & 1.0190 & 1.4328 & 0.038 & 0.962 \\
\hline \multicolumn{9}{|l|}{ Referential process } \\
\hline MIWRAD & 0.4974 & 0.0052 & 0.4980 & 0.0063 & 0.5005 & 0.0079 & 1.245 & 0.295 \\
\hline MIWRRL & 0.5545 & 0.0077 & 0.5530 & 0.0072 & 0.5500 & 0.0070 & 2.006 & 0.143 \\
\hline MH-IWRAD & 0.0037 & 0.0038 & 0.0035 & 0.0034 & 0.0049 & 0.0048 & 0.814 & 0.448 \\
\hline MH-IWRRL & 0.0545 & 0.0077 & 0.0530 & 0.0073 & 0.0500 & 0.0070 & 2.006 & 0.143 \\
\hline IWRAD IWRRL & -0.3128 & 0.5660 & -0.1595 & 0.7191 & 0.0117 & 0.5660 & 1.147 & 0.324 \\
\hline
\end{tabular}

${ }^{*} \mathrm{p}<.05$. MIWRAD, Italian Weighted Referential Activity Dictionary; MIWRRL, Italian Weighted Reflection/Reorganization List; MH-IWRAD, Referential Activity Intensity Index; MHIWRRL, Reflection/Reorganization Intensity Index; IWRAD_IWRRL, Referential Activity and Reflection/Reorganization covariation.

Table 2. Differences in linguistic measures of the RP and LIWIC indexes during the three writing sessions with the high-alexithymia group.

\begin{tabular}{|c|c|c|c|c|c|c|c|c|}
\hline \multirow[t]{2}{*}{ Variable } & \multicolumn{2}{|c|}{ I writing session } & \multicolumn{2}{|c|}{ II writing session } & \multicolumn{2}{|c|}{ III writing session } & \multirow[t]{2}{*}{$\mathbf{F}$} & \multirow[t]{2}{*}{$\mathbf{p}$} \\
\hline & $\mathbf{M}$ & SD & $\mathbf{M}$ & SD & $\mathbf{M}$ & SD & & \\
\hline \multicolumn{9}{|l|}{ LIWIC } \\
\hline I & 4.1664 & 2.7025 & 4.5714 & 2.0623 & 4.5977 & 2.0623 & 0.155 & 0.857 \\
\hline We & 0.5557 & 0.6983 & 0.3807 & 0.6576 & 0.6359 & 0.1763 & 0.244 & 0.785 \\
\hline Affectivity & 7.0236 & 3.5763 & 7.5857 & 3.1244 & 6.2131 & 3.1266 & 0.592 & 0.558 \\
\hline Positive sensations & 4.0771 & 2.3048 & 4.3829 & 3.1637 & 2.7392 & 1.1073 & 1.811 & 0.177 \\
\hline Positive emotions & 1.1843 & 1.0737 & 1.1886 & 1.4528 & 0.7015 & 0.9441 & 0.746 & 0.481 \\
\hline Optimism & 1.5664 & 1.8841 & 2.2600 & 2.2241 & 1.746 & 0.7834 & 1.324 & 0.278 \\
\hline Negative emotions & 2.7857 & 1.7047 & 3.0407 & 1.6931 & 3.1300 & 2.8260 & 0.097 & 0.908 \\
\hline Anxiety & 0.5279 & 0.4817 & 1.1693 & 1.2192 & 1.3185 & 2.0101 & 1.298 & 0.285 \\
\hline Anger & 0.2379 & 0.3374 & 0.3229 & 0.4786 & 0.5377 & 0.7227 & 1.131 & 0.333 \\
\hline Sadness & 1.2443 & 1.7643 & 0.7707 & 0.9227 & 1.0823 & 1.1278 & 0.461 & 0.634 \\
\hline Past time & 1.1379 & 1.4483 & 1.1314 & 1.0807 & 1.4254 & 1.2792 & 0.230 & 0.796 \\
\hline Present time & 10.3771 & 3.8422 & 9.0550 & 3.4173 & 10.4754 & 4.2298 & 0.590 & 0.559 \\
\hline Future time & 0.2264 & 0.4751 & 0.1014 & 0.3795 & 0.2831 & 0.5421 & 0.537 & 0.589 \\
\hline I verbal & 6.1243 & 3.4051 & 5.9021 & 3.9915 & 5.5446 & 3.0115 & 0.093 & 0.911 \\
\hline We verbal & 0.2886 & 0.4155 & 0.6843 & 1.4066 & 0.8415 & 1.0489 & 1.023 & 0.369 \\
\hline \multicolumn{9}{|l|}{ Referential process } \\
\hline MIWRAD2 & 0.4951 & 0.0055 & 0.4953 & 0.0101 & 0.4987 & 0.0085 & 0.766 & 0.472 \\
\hline MIWRRL & 0.5551 & 0.0056 & 0.5513 & 0.0080 & 0.5498 & 0.0067 & 2.120 & 0.134 \\
\hline MHIWRAD2 & 0.0026 & 0.0023 & 0.0019 & 0.0024 & 0.0041 & 0.0056 & 1.229 & 0.304 \\
\hline MHIWRRL & 0.0551 & 0.0056 & 0.0513 & 0.0080 & 0.0498 & 0.0067 & 2.120 & 0.134 \\
\hline IWRAD2 IWRRL & -0.4402 & 0.5196 & -0.2237 & 0.7702 & -0.2441 & 0.6850 & 0.447 & 0.643 \\
\hline
\end{tabular}

${ }^{*} \mathrm{p}<.05$. MIWRAD, Italian Weighted Referential Activity Dictionary; MIWRRL, Italian Weighted Reflection/Reorganization List; MH-IWRAD, Referential Activity Intensity Index; MHIWRRL, Reflection/Reorganization Intensity Index; IWRAD IWRRL, Referential Activity and Reflection/Reorganization covariation. 
LIWIC indexes a significant difference only between the first and third writing sessions, in the direction of an increment in both values in the latter (respectively $\mathrm{p}=.023$; $\mathrm{p}=.010$ ). Moreover, Bonferroni's post hoc analysis revealed for the future time index of LIWIC a significant difference only between the second and third writing sessions $(p=.049)$, in the direction of higher values in the former. No differences in the group of women with high levels of alexithymia emerged in the analysis of the writing process.

As regards differences in the linguistic measures of the RP and LIWIC indexes between the high and low alexithymia groups, several differences emerged. Indeed, the group with low alexithymia scored higher in the LIWIC indexes referring to the future time $(p=.006)$ and sense of "we-ness" (we verbal) indexes $(p=.039)$ and lower in the sadness index $(\mathrm{p}=.049)$ compared to the group with high alexithymia (see Table 3). Moreover, in terms of RP measures, the group with low alexithymia scored higher in MIWRAD2 $(\mathrm{p}=.028)$ and MHIWRAD2 $(\mathrm{p}=.049)$ and lower in IWRAD2 IWRRL $(\mathrm{p}=.041)$ (see Table 3$)$.

In Table $\overline{4}$, two examples of texts produced during the three expressive writing sessions by women with low/high levels of alexithymia were reported in order to show the different linguistic styles.

\section{Discussion}

The literature concerning the role of alexithymia in clinical treatment has so far yielded mixed results. The present work has explored the role played by alexithymia in the emotional process promoted by an EWI, a brief supportive intervention, applied to women undergoing an ART. We have investigated how the alexithymia trait, defined as a multifaceted personality construct, can affect one's ability to self-reflect and self-regulate emotions, hypothesizing that alexithymia may interfere in the emotional and symbolizing process of expressive writing. In fact, when the alexithymia construct is interpreted according to multiple code theory, it can be defined as an emotional disconnection process. Emotional disconnections do not allow a person to associate their bodily and emotional experiences into words. The first objective of this study has been to undertake a linguistic analysis of the writing process intervention during the three sessions of the EWI protocol within low- and high-alexithymia groups. The results revealed that only women with low alexithymia scores manifested a variation in linguistic indexes during the writing sessions, whereas women with high alexithymia scores did not show any differences. These variations could be interpreted as a measure of the emotional and cognitive activation of an elaboration process, activated by writing about stressful events such as infertility or the experience of ART. In particular, women with low alexithymia scores reported a greater use of words expressing affectivity and sadness from the first to the last writing session. The result regarding the increment of sadness words seems to be coherent with previous studies highlighting the importance of expressing negative emotions during the writing process (Smyth, 1998). The capacity to describe the more ambivalent feelings involved in an ART experience can be considered as a meas-

Table 3. Differences in linguistic measures of the RP and LIWIC indexes between the high- and low-alexithymia groups.

\begin{tabular}{|c|c|c|c|c|c|c|}
\hline \multirow[t]{2}{*}{ Variable } & \multicolumn{2}{|c|}{$\begin{array}{l}\text { Low-alexithymia group } \\
\qquad N=21\end{array}$} & \multicolumn{2}{|c|}{$\begin{array}{l}\text { High-alexithymia group } \\
\qquad N=14\end{array}$} & \multirow[t]{2}{*}{$\mathbf{F}$} & \multirow[t]{2}{*}{ p } \\
\hline & M & sd & $\mathbf{M}$ & sd & & \\
\hline \multicolumn{7}{|l|}{ LIWIC } \\
\hline I & 3.8018 & 1.3234 & 4.5443 & 1.2416 & 1.802 & 0.189 \\
\hline We & 0.8004 & 0.5572 & 0.4486 & 0.4056 & 2.440 & 0.128 \\
\hline Affectivity & 6.7004 & 1.6056 & 6.5357 & 1.7571 & 0.057 & 0.813 \\
\hline Positive sensations & 3.9379 & 1.3786 & 2.9971 & 1.6932 & 2.387 & 0.132 \\
\hline Positive emotions & 1.1468 & 0.6863 & 0.6386 & 0.5004 & 3.357 & 0.076 \\
\hline Optimism & 1.6467 & 0.8786 & 1.3643 & 1.0748 & 0.527 & 0.473 \\
\hline Negative emotions & 2.6725 & 1.3238 & 3.4214 & 0.8848 & 1.993 & 0.167 \\
\hline Anxiety & 1.0375 & 0.9050 & 1.0700 & 0.6645 & 0.008 & 0.930 \\
\hline Anger & 0.3254 & 0.3292 & 0.3629 & 0.2597 & 0.078 & 0.782 \\
\hline Sadness & 0.6929 & 0.4768 & 1.0671 & 0.2721 & 3.932 & $0.049 *$ \\
\hline Past time & 1.4157 & 0.8387 & 1.2671 & 0.8618 & 0.198 & 0.659 \\
\hline Present time & 9.9532 & 2.0854 & 9.1071 & 4.0559 & 0.612 & 0.440 \\
\hline Future time & 0.4154 & 0.3378 & 0.0300 & 0.0793 & 8.799 & $0.006^{*}$ \\
\hline I verbal & 4.8043 & 1.3783 & 5.1157 & 3.0635 & 0.167 & 0.686 \\
\hline Weverbal & 0.8857 & 0.8155 & 0.2129 & 0.1969 & 4.599 & $0.039 *$ \\
\hline \multicolumn{7}{|l|}{ Referential process } \\
\hline MIWRAD2 & 0.4981 & 0.0029 & 0.4946 & 0.0055 & 5.278 & $0.028 *$ \\
\hline MIWRRL & 0.5519 & 0.0049 & 0.5549 & 0.2029 & 2.245 & 0.144 \\
\hline MHIWRAD2 & 0.0056 & 0.0026 & 0.0035 & 0.0019 & 4.116 & $0.049 *$ \\
\hline MHIWRRL & 0.0519 & 0.0049 & 0.0549 & 0.0029 & 2.243 & 0.144 \\
\hline IWRAD2_IWRRL & -0.2213 & 0.3272 & -0.5001 & 0.2129 & 4.536 & $0.041^{*}$ \\
\hline
\end{tabular}

${ }^{*}$ p $<.05$. MIWRAD, Italian Weighted Referential Activity Dictionary; MIWRRL, Italian Weighted Reflection/Reorganization List; MH-IWRAD, Referential Activity Intensity Index; MHIWRRL, Reflection/Reorganization Intensity Index; IWRAD_IWRRL, Referential Activity and Reflection/Reorganization covariation. 
ure of increment in awareness. In fact, during ART, a major difficulty in accepting one's infertility, alongside conflictual emotions related to the desire/frustration to generate a child, are implicit. Within the group of women with low alexithymia, another relevant finding associated with the process activated by the expressive intervention is linked to the increment in the use of verbs declined in the future tense. This increment was observed in the second writing session. Indeed, reporting more issues associated with what will happen in the future is an indirect index of the use of imagination related to the pregnancy and the future birth. As the literature on alexithymia indicates, one of the main difficulties faced by people with high alexithymia is a lack of ability to imagine or prefigure the future, revealing reduced imaginative capacities, expressed in a paucity of fantasies as regards both their frequency and their vividness. This difficulty also implies the failure in using fantasy in the affect regulation process (Goerlich, 2018; Luminet et al., 2018; Lumley, Neely, \& Burger, 2007). In the present study, this difficulty seemed to be confirmed by the observation of the activation of different processes in two groups, with the group with higher alexithymia failing to show any activation in imag- ining the future. These findings seem to confirm the hypothesis that alexithymia can interfere with the emotional and symbolizing processes of expressive writing (Lumley, 2004; Paez et al., 1999; Silva, Vasco, \& Watson, 2018).

In contradistinction to our hypothesis, significant variations within the groups in the RP linguistic measures during the three sessions of writing did not emerge. Nevertheless, when analyzing differences between the groups with low/high alexithymia in terms of RP linguistic measures in the total written production, some important findings emerged. Certainly, the low-alexithymia group reported higher values in the IWRAD and MHIWRAD indexes compared to the high-alexithymia group, thereby showing greater abilities in symbolizing. On the other hand, the low IWRAD, MH-IWRAD and IWRAD_IWRRL covariation indexes reported by the high-alexithymia group seemed to indicate the presence of a difficulty in being involved in the writing process and a lack of symbolizing process. This finding seems to be in line with our second aim as well as with the presence of a disconnection pattern in the high-alexithymia group. This also enriches the literature highlighting EWI supportive function in promoting the emotional elaboration and

Table 4. Clinical examples of EWI in two subjects with different levels of alexithymia.

Subject in the high-alexithymia group (alexithymia total score = 64; IWRAD: 0.482 - high-value)

Time 1: All'inizio hai tanto entusiasmo e poi quando fai il test ti senti come vuota. [At the beginning you have a lot of enthusiasm and then when you take the test you feel empty.]

Time 2: Dopo tre volte spero che vada bene anche perché mi sento un po' stanca e stressata. All'inizio avevo tanto entusiasmo, adesso un po' meno. [After three times I hope it will be successful, because I also feel a little tired and stressed. At the beginning I had a lot of enthusiasm, but now a little less.]

Time 3: Ripensando al passato rimpiango di non essermi controllata prima così avrei potuto prevenire e fermare meglio la mia malattia. Magari sarei diventata mamma prima e avrei fatto contenti un po' tutti. Adesso mi sento sempre un po' più indietro rispetto alle mie amiche e cugine già mamme. Certe volte mi sento un po' estranea anche a tutti i discorsi che fanno, anche se adesso anche loro hanno capito e si parla meno di bimbi e gravidanze. [Looking back on the past, I regret not checking myself sooner in order to better prevent and stop my disease. Maybe I would have become a mother sooner and I would have made everyone happy. Now I always feel a little behind compared to my friends and cousins who are already mothers. Sometimes I feel a little foreign even to all the speeches they make, even if now they understand and we talk less about babies and pregnancies.]

Subject in the low-alexithymia group (alexithymia total score = 33; IWRAD: 0.502 - high-value)

Time 1: Non pensavo sarebbe mai accaduto. È una di quelle classiche cose che si pensa accadono solo agli altri. Mi sono sentita sbagliata, punita ingiustamente, pensando a chi figli non ne vuole e rimane incinta inconsapevolmente. Sono fiduciosa, ho accanto un compagno che mi è vicino, mi sostiene, è molto ottimista. Ne ho parlato serenamente con i miei amici più cari e la mia famiglia. Oggi sono qui sperando di poter iniziare, potrebbe quindi essere l'inizio di un cammino di speranze, o la conferma di non potere neanche iniziarlo. [I didn't think it would ever happen. It's one of those classic things that a person thinks that can only happen to others. I felt wrong, unjustly punished, thinking about people who do not want children and become unwittingly pregnant. I am confident, I have a partner next to me who is close to me, he supports me, he is very optimistic. I talked serenely about the situation with my closest friends and with my family. Today I am here hoping to be able to start the procedure. It could therefore be the beginning of a journey of hope, or the confirmation that I cannot even begin it.]

Time 2: Oggi siamo più felici perché abbiamo potuto iniziare questo percorso, e tra meno di un mese sapremo se ce l'abbiamo fatta! Sono molto serena perché mio marito mi è sempre accanto, sereno dolce, disponibile, come e più del solito. Ieri siamo andati a trovare una nostra cara amica che ha appena partorito.. è stata una bellissima sensazione che spero di vivere presto! [Today we are happier because we were able to start this process, and in less than a month we will know if we did it! I am very serene because my husband is always by my side, serene sweet, available, even more than usual. Yesterday we went to find a dear friend of ours who has just given birth ... it was a wonderful feeling that I hope to live out soon!]

Time 3: è passata una settimana dall'inizio delle terapie.. e oggi spero di sentire buone notizie, che i follicoli sono diventati più grandi così da avere la certezza che la prossima settimana possiamo effettuare il pick-up. Sono tranquilla, i medicinali non mi stanno causando effetti collaterali, sono serena e speranzosa. Ho accanto un uomo meraviglioso e pieno di fiducia, desideriamo tanto questo bambino.. .abbiamo il nome pronto da mesi e speriamo davvero tanto di poterlo abbracciare presto! [A week has passed since the beginning of the therapy ... and today I hope to hear good news, that the follicles have become bigger so that we can be sure that we can pick up them next week. I am calm, the medicines are not causing me side effects, I am calm and hopeful. I have a wonderful and confident man next to me, we really want this baby ... we have had the name ready for months and we really hope to be able to hug him soon!] 
self-regulation process (Kupeli et al., 2019). In fact, one of the plausible theories about the processes underlying EWI is the emotional regulation process, which takes into account the role of mastery in managing emotions, physiological responses and behaviours associated with the difficult experiences one faces (Kupeli et al., 2019; Lepore, Greenberg, Bruno, \& Smyth, 2002). According to this interpretation, the therapeutic element of emotional arousal following expressive disclosure is the important component: disclosure functions have an affect regulation process, with the act of confronting thoughts and feelings associated with trauma improving perceptions of control and self-efficacy over the negative emotions experienced as a result of adversity (Kupeli et al., 2019).

The qualitative differences between the groups in RP can be seen in the clinical exemplification (Table 4). The women's writings showed a prototypical difference in linguistic style. In writings related to a subject with low alexithymia, the women were able to involve themselves in the experience and the reader could easily understand their ambivalence and contrasting feelings connected to it. In the writings related to a case with high alexithymia, the women manifested a disconnection pattern, being unable to describe and become involved in the experience, using generic and poor sentences.

As regards LIWC, the analysis between the groups indicated that the low-alexithymia group reported a lower use of words expressing sadness and a higher use of future perspectives and "we verbal" compared to the high-alexithymia group. These results seem to be congruent with the hypothesis that high alexithymia implies an over-estimation of negative feelings (i.e. any emotional variation can be read as a negative emotion). Moreover, low scores in "we verbal" may represent a signal of lower abilities in thinking about the experience as a couple (being involved in a sense of "we-ness"), whereas low scores in future appear to express a concrete way of thinking, with difficulties in using the imagination process.

The results of this preliminary study need to be interpreted in the light of some limitations. First, the small size of the sample represented a constraint; future studies should thus incorporate a larger number of subjects. A second limitation was the inclusion of only female participants; future studies should also include male subjects. Third, the enrolment of participants undergoing an invasive medical treatment, which represents a specific stressful situation, rendered it difficult to distinguish primary from secondary alexithymia, where secondary alexithymia is posited to arise as a consequence of events occurring later in life as a defence mechanism in an attempt to cope with a difficult situation (Messina et al., 2014). Fourth, the impossibility of realizing more distanced writing sessions was a limitation, as it implied completing the writing protocol after several critical points of the medical procedure (such as oocyte retrieval, embryo transfer or the pregnancy test) that would have strongly conditioned the findings. A future study should propose three different writing instructions, one for each session, in order to widely explore the issue. Fifth, the participants with fertility problems were recruited from just one infertility clinic in Rome, which may have introduced a selection bias. Sixth, the use of a self-report questionnaire for the assessment of alexithymia levels engendered some constraints, so future studies should use additional instruments such as the Toronto Structured Interview for Alexithymia (Bagby, Taylor, Parker, \& Dickens, 2006) a semi-structured interview for the evaluation of the construct that allows for a more accurate assessment of the dimension.

\section{Conclusions}

Despite these limitations, the findings of the present study showed that in this specific field of application, EWI seemed to promote an emotional process aimed at self-regulating and symbolizing the experience in women with low levels of alexithymia, whereas the same intervention did not yield significant findings in the high-alexithymia group. This difference may have owed to the presence of disconnection patterns between subsymbolic and symbolic systems in subjects with greater difficulties in emotional regulation than in high-alexithymia cases. This finding appears to confirm the results of previous investigations that have shown that subjects with high alexithymia do not appear to reap the same positive effects after writing (Di Trani et al., 2014; Lumley, 2004; Pennebaker \& Chung, 2007; Solano, 2013).

Therefore, these findings suggest that evaluating alexithymia levels before the implementation of EWI may be useful in order to address the intervention to people who can benefit from it or to set it based on the capabilities or difficulties manifested by the patient in question. In this light, a previous evaluation of alexithymia levels may allow researchers to implement certain strategies capable of overcoming emotional disconnections (Bucci, 1997b). In this direction, according to multiple code theory specific precautions in order to overcoming disconnection in people with high alexithymia may be a greater number of writing sessions as well as the use of an input focused on body experience or symptomatology (Kupeli et al., 2019). These results need to be confirmed in future studies and the role of alexithymia in EWI must be further investigated while additionally including an evaluation of other relevant dimensions, such as stress levels, psychological symptomatology and specific anamnestic information.

\section{References}

Bagby, R.M., Parker, J.D.A., \& Taylor, G.J. (1994). The twenty item Toronto Alexithymia Scale-I: Item selection and cross validation of the factor structure. Journal of Psychosomatic Research, 38, 23-32.

Bagby, R.M., Taylor, G.J., Parker, J.D., \& Dickens, SE. (2006). The development of the Toronto Structured Interview for 
Alexithymia: Item selection, factor structure, reliability and concurrent validity. Psychotherapy and Psychosomatics, 75, 25-39.

Ben Shlomo, S., Skvirsky, V., Taubman-Ben-Ari, O., Azuri, Y., \& Horowitz, E. (2016). Mental health of women entering fertility treatment: What role do age and internal resources play? Stress and Health, 33, 470-476. doi:10.1002/smi.2733

Boivin, J. (2003). A review of psychosocial interventions in infertility. Social Science \& Medicine, 57, 2325-2341. doi:10.1016/S0277-9536(03)00138-2

Bressi, C., Taylor, G.J., Parker, J.D., Bressi, S., Brambilla, V., Aguglia, E., ... Invernizzi, G. (1996). Cross validation of the factor structure of the 20-item Toronto Alexithymia Scale: An Italian multicenter study. Journal of Psychosomatic Research, 41, 551-559. doi:10.1016/S0022-3999(96) 00228-0

Bucci, W. (1997a). Psychoanalysis and cognitive science: A multiple code theory. Guilford Press.

Bucci, W. (1997b). Symptoms and symbols: A multiple code theory of somatization. Psychoanalytic Inquiry, 17, 151172. doi:10.1080/07351699709534117

Bucci, W. (2001). Pathways of emotional communications. Psychoanalytic Inquiry, 20, 40-70. doi:10.1080/0735169210 9348923

Bucci, W., Kabasakalian, R., \& the Referential Activity Research Group (2004). Instructions for scoring Referential Activity (RA) in transcripts of spoken narrative texts. Available from: http://www.thereferentialprocess.org

Bucci, W., \& Maskit, B. (2007). Beneath the surface of the therapeutic interaction: The psychoanalytic method in modern dress. Journal of the American Psychoanalytic Association, 55, 1355-1397. doi:10.1177/000306510705500412

Bucci, W., Maskit, B., \& Murphy, S. (2015). Connecting emotions and words: The referential process. Phenomenology and the Cognitive Sciences, 15, 359-383. doi:10.1007/ s11097-015-9417-z

Campbell, R.S., \& Pennebaker, J.W. (2003). The secret life of pronouns: Flexibility in writing style and physical health. Psychological Science, 14, 60-65.

Conrad, R., Schilling, G., Langenbuch, M., Haidl, G., \& Liedtke, R. (2001). Alexithymia in male infertility. Human Reproduction, 16, 587-592. doi:10.1093/humrep/16.3.587.

Di Trani, M. (2007). Per chi? Il ruolo delle variabili individuali sugli effetti della tecnica della scrittura [For whom? The role of individual dimensions on the effect of the expressive writing intervention]. In L. Solano (Ed.), Scrivere per pensare [Writing for thinking] (pp. 39-64). Franco Angeli Editore.

Di Trani, M., Mariani, R., Renzi, A., Greenman, P. S., \& Solano, L. (2018). Alexithymia according to Bucci's multiple code theory: A preliminary investigation with healthy and hypertensive individuals. Psychology and Psychotherapy: Theory, Research and Practice, 91, 232-247. doi:10.1111/papt.12158

Di Trani, M., Renzi, A., \& Solano, L. (2014). Elaborare le esperienze difficili scrivendo. La tecnica della scrittura di James Pennebaker [Processing difficult experience through writing: James Pennebaker's writing technique]. Nuove Arti Terapie, 24, 95-109. Available from: https://www.nuoveartiterapie. net/new/pdf/rivista/23-24-NuoveArtiTerapie.pdf

Dumitru, R.T., \& Turliuc, M.N. (2015). Predictors of stress among infertile couples. Revista de Psihologie, 61, 268-282.

Edwards, E.R., Shivaji, S., Micek, A., \& Wupperman, P. (2020). Distinguishing alexithymia and emotion differentiation conceptualizations through linguistic analysis. Personality and Individual Differences, 157, e109801.
Frederiksen, Y., O’Toole, M. S., Mehlsen, M. Y., Hauge, B., Elbaek, H. O., Zachariae, R., \& Ingerslev, H. J. (2017). The effect of expressive writing intervention for infertile couples: A randomized controlled trial. Human Reproduction, 32, 391-402. doi:10.1093/humrep/dew320

Galhardo, A., Cunha, M., \& Pinto-Gouveia, J. (2011). Psychological aspects in couples with infertility. Sexologies, 20, 224-228. doi:10.1016/j.sexol.2011.08.005

Goerlich, K.S. (2018). The multifaceted nature of alexithymia - A neuroscientific perspective. Frontiers in Psychology. doi:10.3389/fpsyg.2018.01614

Gourounti, K., Anagnostopoulos, F., Griva, F., \& Vasmatzis, G. (2016). Alexithymia and fertilityrelated stress. Women \& Health, 56, 312-325. doi:10.1080/03630242.2015.1088119

Grabe, H.J., Frommer, J., Ankerhold, A., Ulrich, C., Groger, R, Franke, G.H., ... Spitzer C. (2008). Alexithymia and outcome in psychotherapy. Psychotheraphy and Psychosomatics, 77,189-194. doi:10.1159/000119739

Grynberg, D., Chang, B., Corneille, O., Maurage, P., Vermeulen, N., Berthoz, S., \& Luminet, O. (2012). Alexithymia and the processing of Emotional Facial Expressions (EFEs): Systematic review, unanswered questions and further perspectives. PLoS ONE, 7, e42429. doi:10.1371/journal.pone.0042429

Klein, K., \& Boals, A. (2001). Expressive writing can increase working memory capacity. Journal of Experimental Psychology: General, 130, 520-533.

Kupeli, N., Chatzitheodorou, G., Troop, N. A., McInnerney, D., Stone, P., \& Candy, B. (2019). Expressive writing as a therapeutic intervention for people with advanced disease: a systematic review. BMC Palliative Care, 18, 65. doi:10.1186/s12904-019-0449-y

Lamas, C., Chambry, J., Nicolas, I., Frydman, R., Jeammet, P., \& Corcos, M. (2006). Alexithymia in infertile women. Journal of Psychosomatic Obstetrics \& Gynecology, 27, 23-30. doi:10.1080/01674820500238112.

Lepore, S.J., Greenberg, M.A., Bruno, M., \& Smyth, J.M. (2002). Expressive writing and health: self-regulation of emotion-related experience, physiology, and behavior. In: S.J. Lepore and J.M. Smyth (Eds.), The writing cure: How expressive writing promotes health and emotional wellbeing (pp. 99-117). American Psychological Association.

Luminet, O., Bagby, R.M., \& Taylor, G.J. (2018). Alexithymia advances in research, theory, and clinical practice. Cambridge University Press.

Lumley, M.A. (2004). Alexithymia, emotional disclosure, and health: A program of research. Journal of Personality, 72, 1271-1300.

Lumley, M.A., Neely, L.C., \& Burger, A.J. (2007). The assessment of alexithymia in medical settings: Implications for understanding and treating health problems. Journal of Personality Assessment, 89, 230-246. doi:10.1080/00223 890701629698

Mariani, R., Di Trani, M., Negri, A., \& Tambelli R. (2020). Linguistic analysis of autobiographical narratives in unipolar and bipolar mood disorders in light of multiple code theory. Journal of Affective Disorders, 273, 24-31. doi: 10.1016/ j.jad.2020.03.170

Mariani, R., Maskit, B., Bucci, W., \& De Coro, A. (2013). Linguistic measures of the referential process in psychodynamic treatment: The English and Italian versions. Psychotherapy Research, 23, 430-447. doi:10.1080/10503307.2013.794399

Matthiesen, S., Klonoff-Cohen, H., Zachariae, R., Jensen-Johansen, M.B., Nielsen, B.K., Frederiksen, Y., ... Ingerslev, 
H.J. (2012). The effect of an expressive writing intervention (EWI) on stress in infertile couples undergoing assisted reproductive technology (ART) treatment: A randomized controlled pilot study. British Journal of Health Psychology, 17, 362-378. doi:10.1111/j.2044-8287.2011.02042.x

Maskit, B. (2014). The Discourse Attributes Analysis Program (DAAP) operating instructions. Figshare. doi:10.6084/m9. figshare. 947740

Maskit, B., Bucci, W., \& Murphy, S. (2012). Computerized language measures. The Referential Process. Available from: https://sites.google.com/site/referentialprocess/dictionarymeasures-and-computer-programs

Maskit, B., \& Murphy, S. (2011). The Discourse Attributes Analysis Program. The Referential Process. Available from: http://www.thereferentialprocess.org/the-discourse-attributes-analysis-program-daap.

Messina, A. Beadle, J.N., \& Paradiso, S. (2014). Towards a classification of alexithymia: Primary, secondary and organic. Journal of Psychopathology, 20, 38-49.

Moreno-Rosset, C., del Castillo Aparicio, M., Ramírez-Uclés, I., \& Martín Díaz, M.D. (2011). Estados emocionales y afectivos en infertilidad: Un estudio comparativo con personas fértiles. Behavioral Psychology, 19, 683-703.

Negri, A., Esposito, G., Mariani, R., Savarese, M., Belotti, L., Squitieri, B., \& Bucci, W. (2018). The Italian weighted reflection and reorganization list (I-WRRL): A new linguistic measure detecting the third phase of the referential process. Research in Psychotherapy: Psychopathology, Process and Outcome, 21, 5-6.

Nemiah, J. C., Freyberger, H., \& Sifneos, P. E. (1976). Alexithymia: A view of the psychosomatic process. In O.W. Hill (Ed.), Modern trends in psychosomatic medicine (Vol. 3, pp. 430-439). Butterworths.

Paez, D., Velasco, C., \& Gonzalez, J. L. (1999). Expressive writing and the role of alexythimia as a dispositional deficit in self-disclosure and psychological health. Journal of Personality and Social Psychology, 77, 630-641.

Panagopoulou, E., Montgomery, A., \& Tarlatzis, B. (2009). Experimental emotional disclosure in women undergoing infertility treatment: Are drop-outs better off? Social Science \& Medicine, 69, 678-681. doi:10.1016/j.socscimed.2009.06.031

Pedrosa Gil, F., Ridout, N., Kessler, H., Neuffer, M., Schoechlin, C., Traue, H.C., \& Nickel, M. (2009). Facial emotion recognition and alexithymia in adults with somatoform disorders. Depression and Anxiety, 26, 26-33. doi:10.1002/da.20456

Pennebaker, J.W. (1997). Writing about emotional experiences as a therapeutic process. Psychological Science, 8, 162-166. doi:10.1111/j.1467-9280.1997.tb00403.x

Pennebaker, J.W., Booth, R.J., \& Francis, M.E. (2007). Operator's manual: Linguistic inquiry and word count: LIWC2007. LIWC.net.

Pennebaker, J.W., \& Chung, C. K. (2007). Expressive writing, emotional upheavals, and health. In H.S. Friedman \& R.C. Silver (Eds.), Foundations to health psychology (pp. 263284). Oxford University Press.

Pennebaker, J.W., Mayne, T.J., \& Francis, M.E. (1997). Linguistic predictors of adaptive bereavement. Journal of Personality and Social Psychology, 72, 166-183.

Pennebaker, J.W., Mehl, M.R., \& Niederhoffer, K.G. (2003). Psychological aspects of natural language use: Our words, ourselves. Annual Review of Psychology, 54, 547-577.

Petrie, K.P., Booth, R.J., \& Pennebaker, J.W. (1998). The immunological effects of thought suppression. Journal of Personality and Social Psychology, 75, 1264-1272. Porcelli, P., Bagby, R.M., Taylor, G.J., De Carne, M., Leandro, G., \& Todarello, O. (2003). Alexithymia as predictor of treatment outcome in patients with functional gastrointestinal disorders. Psychosomatic Medicine, 65, 911-918.

Ramírez-Uclés, I., Castillo-Aparicio, M., \& Moreno-Rosset, C. (2015). Psychological predictor variables of emotional maladjustment in infertility: Analysis of the moderating role of gender. Clínica y Salud, 1, 57-63. doi:10.1016/j.clysa. 2015.01.002

Renzi, A., Solano, L., Di Trani, M., Ginobbi, F., Minutolo E., \& Tambelli, R. (2019). The effects of an expressive writing intervention on pregnancy rates, alexithymia and psychophysical health during an assisted reproductive treatment. Psychology \& Health. doi:10.1080/08870446.2019.1667500

Ridout, N., Thom, C., \& Wallis, D.J. (2010). Emotion recognition and alexithymia in females with non-clinical disordered eating. Eating Behaviors, 11, 1-5. doi:10.1016/j.eatbeh. 2009.07.008

Rohrbaugh, M.J., Shoham, V., Skoyen, J. A., Jensen, M., \& Mehl, M.R. (2012). We-talk, communal coping, and cessation success in a couple-focused intervention for healthcompromised smokers. Family Process, 51, 107-121. doi:10.1111/j.1545-5300.2012.01388.x

Shi, X.B., Wu, P.L. \& Zhu, X.Z. (2006?). Alexithymia and its features in patients with infertility. Chinese Journal of Clinical Psychology, 14, 403-404. Available from: http://caod. oriprobe.com/articles/10695620/Alexithymia_and_Its_Feature_in_Patients_with_Infertility.htm

Silva, A., Vasco, A., \& Watson, J. (2018). Alexithymia and emotional processing: A longitudinal mixed methods research. Research in Psychotherapy: Psychopathology, Process and Outcome, 21(1). https://doi.org/10.4081/ripppo.2018.292

Smyth, J.M. (1998). Written emotional expression: Effect sizes, outcome types, and moderating variables. Journal of Consulting and Clinical Psychology, 66,174-184.

Solano, L. (2013). Lo psicologo ovunque possibile [The psychologist everywhere]. In L. Solano (Ed.), Tra mente e corpo [Between mind and body] (pp. 485-516). Raffaello Cortina Editore.

Solano, L., Donati, V., Pecci, F., Persicheeti, S., \& Colaci, A. (2003). Post-operative course after pailloma resection: Effects of written disclosure of the experience in subjects with different alexithymia levels. Psychosomatic Medicine, 65, 477-484.

Spera, S.P., Buhrfeind, E.D., \& Pennebaker, J.W. (1994). Expressive writing and coping with job loss. Academy of Management Journal, 37, 722-733.

Tausczik, Y. R., \& Pennebaker, J. W. (2010). The psychological meaning of words: LIWC and computerized text analysis methods. Journal of Language and Social Psychology, 29, 24-54.

Taylor, G.J. (2004). Alexithymia: 25 years of theory and research. In I.L. Temoshok \& A. Vingerhoets (Eds.), Emotional expression and health (pp. 137-153). Brunner-Routledge.

Taylor, G.J., \& Bagby, R.M. (2013). Psychoanalysis and empirical research: The example of alexithymia. Journal of the American Psychoanalytic Association, 61, 99-133. doi:10.1177/0003065112474066

Taylor, G.J., Bagby, R.M., \& Parker, J.D.A. (1997). Disorders of affect regulation: Alexithymia in medical and psychiatric illness. Cambridge University Press. 